\title{
Safe Contact Lens Practices during Coronavirus (COVID-19) Pandemic
}

\section{Sanjeev Bhattarai}

Assistant Professor, Department of Ophthalmology, Maharajgunj Medical Campus, Institute of Medicine, Maharajgunj, Kathmandu, Nepal

\section{ABSTRACT}

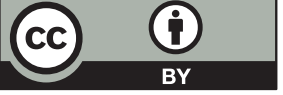

This work is licensed under a Creative Commons Attribution 4.0 Unported License.

World is threatened by different variants of Coronavirus to an extent that life has been jeopardized in all services including eye care facility. Contact lenses which are one of the preferred modes of vision correction are not an exception to COVID-19 infection. Recently there is confusion among practitioners and patients regarding the safety of contact lens use during COVID-19. This review article summarizes the current recommendations and findings regarding contact lens use during COVID-19. Though eye health professionals and contact lens users are at risk to contract Coronavirus, with proper care, maintenance and disinfection techniques, it would be safe to continue wearing contact lenses. There is currently no strong evidence to suggest that contact lenses are not safe to wear during COVID 19 times. Eye care practitioners responsible for contact lens application must wear special surgical masks, protective glasses or visors and disposable waterproof gloves. Regarding soft contact lenses, preferably daily disposable lenses should be used from the sterile packages. Similarly for rigid gas permeable lenses, lenses made up of fluoropolymer contained in sealed packages must be motivated for the individuals. Since the infected hands can bring the virus to the eyes, contact lens users should avoid touching the nose, mouth and eye unnecessarily. Contact lens after each use must be disinfected with hydrogen peroxide solutions with a special catalyst container. In the case of eye redness and Flu like symptoms, contact lens trial and wearing practices should be suspended or postpone till the condition becomes normal. To minimize the contact time between the patient and examiner soft copy of the instruction sheet, teleconsultation should be motivated. Though the eye is an unlikely site for Coronavirus infection, eye doctors should warn the contact lens wearers to follow scrupulous contact lens hygiene rules.
\end{abstract}

\section{KEY WORDS}

Conjunctivitis, Contact lenses, COVID-19, Disinfection, Hydrogen peroxide, Tears, Teleconsultation

\section{INTRODUCTION}

Contact lens is a very effective and safe way to correct vision. Nowadays, more than 140 millions of people worldwide are using various designs and modalities of contact lenses. 'It is a thin, optical device that rest on the surface of the cornea to correct the refractive error of an individual. Recently there is a usual question "Does contact lenses wear has any risk of getting infection with COVID-19?" So, this article is focused on answering this question with the help of various published literature review along with proper care and maintenance of contact lenses during this pandemic of Coronavirus.

\section{CURRENT UNDERSTANDINGS AND RECOMMENDATIONS}

According to Michaud, the first element to consider is the lens itself. ${ }^{2}$ According to him, no any study has suggested that in an ocular surface, the virus can colonize the lens and remain there. So the contact lens could not be the vector for the transmission of the disease. The possibility of transmission of disease can be further reduced by using daily disposable lenses instead of using yearly disposable lenses. Such daily disposable lenses could be safest for eye health and hygiene. The second element is lens handling, where there occurs eye and lens interaction for insertion and removal of the lens. In such pandemic, proper hand washing during lens wear could play a significant role for reduction of spread of disease. The third element, the lens care which should be performed with lens products and accessories recommended by eye care professionals. To minimize a contact lens infection, a hydrogen peroxide

\footnotetext{
*Corresponding Author | Dr.Sanjeev Bhattarai, BOptom, O.D., M.Optom. BP Koirala Lions Center for Ophthalmic Studies, Maharajgunj, Kathmandu, Nepal. Email: bhattarai_sanjeev@yahoo.com I
}

Contact Number: + 9779841509323. 
solution should be used for disinfection and overnight soaking in multipurpose contact lens solution. Before insertion of contact lenses in morning, it should be rinsed well with a saline solution formulated for contact lenses. ${ }^{2}$

A Chinese Ophthalmologist was one of the first victims of COVID-19 after being exposed to an asymptomatic patient. ${ }^{2}$ Afterwards, it was suspected that there might be presence of the COVID-19 virus in the eyes and transmitted through tears. Somehow the issue rose regarding the potential risk of wearing contact lenses during the pandemic. Michaud conclusion came from a literature review published in April 22, 2020, based on nearly 200 peer reviewed articles. Some of these articles reported the ability of the virus to bind to cells on the ocular surface. But such binding might not be able to generate an eye infection. Wearing contact lenses remains safe as long as patient follow the required hygienic measures. Due to frequent blinking and exchange of tears, the virus is unlikely to stay on the eye surface long enough to cause ocular problems. A trace of these viruses has been found in the tears of patients but direct infection or transmission through the ocular surface has not been documented. Less than one percent of infected people might develop eye irritation in the form of acute red eye. Such red eye condition can last 10 to 20 days after the first symptom related to virus appear. In few cases cornea may also be affected and such ocular condition can be easily treated with usual treatments without other long term ocular complication. ${ }^{2}$

Similarly, a review article by Olivares - de Emparan et.al has summarized that COVID-19 can also cause conjunctivitis (red eye) and can therefore theoretically be transmitted by contact with tears and other ocular secretions. ${ }^{3}$ Hersh suggested that there are some risk factors for Corona virus infection due to wear of contact lenses. Contact lenses may cause eye irritation and wearers tend to touch their eyes more often than spectacles wearers. The novel Coronavirus can enter our body through our eyes in addition to nose and mouth. ${ }^{4}$ When someone who has COVID-19 sneezes, coughs, or even talks, they spread droplet that contain the virus. The surrounding people are most likely to breathe in those droplets, but the virus can also enter their body through their eyes too. Another way we can contract the virus is if the virus lands on our hand or fingers and we then touch our nose, mouth, or eyes. However, this is less common. There is currently no evidence to prove that wearing contact lenses increases your risk of contracting the new corona virus. Morgan advised that washing our hands thoroughly especially after touching surfaces can help keep us safe. ${ }^{5}$ According toYong et.al. , we can get COVID-19 by touching a surface contaminated with it and then touching our eyes without washing hands. This could raise our risk. ${ }^{6}$
Kramer has advised to wear surgical masks for protection of mouth and nose in all clinical settings until further notice. ${ }^{7}$ According to Kramer, for disinfection of soft contact trial lenses, it should be done with 3\% hydrogen peroxide solution followed by complete neutralization with $0.9 \%$ sodium chloride solution for 15 minutes or lens can be stored overnight in isotonic saline. It needs four hours for complete neutralization. Hydrogen peroxide (3\%) is highly acidic with $\mathrm{PH}$ of 3-4. For rigid gas permeable lenses, disinfection of trial lenses can be done by storing the lenses overnight or 4 to 6 hours in multipurpose solution formulated for RGP lenses.

Similarly, United States Centers for Disease Control and Prevention (CDC) suggested that there is no evidence that contact lens user group is more at risk of contracting Coronavirus than glasses wearers. ${ }^{8}$ There have been no confirmed cases of COVID-19 transmission through handling of contact lenses. According to CDC, it is of great importance to follow the following healthy habits when wearing contact lenses to help protect our eyes, especially in this pandemic.CDC guidelines for care and maintenance of contact lenses specially during COVID-19 is further supported by other few published literatures too. ${ }^{9-11}$ As per their recommendation, before handling contact lenses, both hands should be washed for at least 20 seconds with soap and water and dry with clean paper or cloth. The contact lenses should be cleaned by rubbing it with multipurpose contact lens solution and rinsed with it. Fresh solution should be used every time to disinfect and store contact lenses. The contact lens case or kit should be replaced at least once every 3 months. In cases of daily disposable lenses, it should be disposed after each wear. For reusable lenses, it should be cleaned and disinfected according to eye care professionals. When some one is having illness, he/she should stop contact lens wear until healthy again. Similarly contact lenses should be removed from eyes when we are taking showers and before going swimming. The contact lens user should avoid rubbing or touching their eyes during contact lens wear. But if it is needed, protective eye shields or any suggested devices should be used along with contact lenses. The contact lens user should avoid rubbing or touching their eyes during contact lens wear. Consultation with an Optometrist or Ophthalmologist is required in cases of any unusual signs and symptoms.

Similarly, Jones et al. mentioned that in some instances of direct contact with the patients, greater protection from N95 masks can be achieved. Additionally, clinician should wear gloves whenever they are in direct contact with patient's body fluids and secretions. ${ }^{12}$ They have advised to wear a face covering both by patient and clinician and contact lens application and removal technique should be done in a socially distanced way. Clinician should use personal 
protective equipment (PPE). Recently practices have now made adaptations that make close contact safer. These include using clear plastic screens to separate practitioner and patient during application and removal teaching and using videos that help show patient how to handle their lenses. A large breath shields should be added to slit lamp biomicroscope to reduce droplet spread. Only sealed and sanitizes blisters should be used for trials every time and discarded used lenses. According to American Academy of Optometry, specialty products like rigid gas permeable (RGP) lenses, Scleral lenses, Ortho-K lenses trial sets should be disinfected using $3 \%$ hydrogen peroxide. For hard contact lenses, trial lenses must be stored dry for a week before trying on the next patient. Similarly, to minimize contact time between patient and examiner soft copy of the instruction sheet can be forwarded to the new patients and follow up examinations should be done only if they are an absolute must. Tele consulting may be tried, and the patients can be taught to take the photograph of the eye with lens on way of instructional video. ${ }^{13}$

\section{WAYS FORWARD}

To be in safe ophthalmic practices, all the clinicians should wear personal protective equipment(PPE) including gloves ,masks, face and eye shields, caps while handling contact lens users as few literatures have warned that human tears and ocular discharge during face to face communication and close examination of the patients may transmit the Coronavirus. ${ }^{14,15}$ Moreover directly touching the patient eyelids, corneal and conjunctiva in an unsafe manner may be a clinical factor for entry of the virus to healthy individuals.

CONCLUSION: Majority of the literatures suggested that there is no significant evidence that wearing contact lenses increases our risk of Coronavirus infection. With prompt care and maintenance, contact lenses are safe to wear during COVID-19 times. The contact lens clinic should function as before the crisis of the pandemic. Hand washing with soap and water, contact lens hygiene including the work surface disinfection are always a part of safe practices in handling contact lenses. These are even more important in the COVID-19 pandemic times.

\section{CONCLICT OF INTEREST : None.}

\section{REFERENCES::}

1.Denniston A, Murray P. Oxford Handbook of Ophthalmology. First Ed. New York: Oxford University Press; 2006:626.

2. Michaud L. Does wearing contact lenses put you at greater risk of getting COVID-19? THE CONVERSATION. Google (Online June 18, 2020).

3.Olivares-de Emparan JP, SardiCorrea C, Lopez-ulloa JA,
Viteri JS, Penniecook JA, Jimenez-Roman J. COVID-19 and the eye: how much do we really know? A best evidence review. Oftalmologia, 2020; 83(3):250-61. DOI:10.5935/00042749.20200067

4. Hersh E. Can wearing contact lenses increase your risk of COVID-19? Healthline. Google (Online June 8,2020)

5.Morgan P. Contact Lens wears during COVID19 pandemic. Cont Lens Anterior Eye 2020; 43:213.

6.Yong Yu, Ruixue Tu and Xu Shao et al. A comprehensive Chinese experience against SARS-CoV-2 in ophthalmology. Eye and Vision (2020) 7:19 https://doi.org/10.1186/s40662020-00187-2

7. Elise K. Contact lens practice in the time of COVID-19. Healio Optometry. Online on July 23, 2020.

8. CDC guidelines for contact lens wear during COVID19. Available from: https://www.cdc.gov/contactlenses/index. html. [Last accessed on 2020 Jun 24]

9 BCLA: Contact Lens Wear and corona virus (COVID-19) guidance 2020. The new guidance from NHS England.

10. Boyd K. How to take care of contact lenses. American Academy of Ophthalmology. Online on March 04, 2021

11. Estopa MV, Wolffsohn JS,Beukes E, Trott M,Smith L, Allen PM. Soft contact lens wearer's compliance during the COVID-19 pandemic. Cont Lens Anterior Eye. 2020 Aug 14; 101359 doi: 10.1016/j.clae.2020.08.003.

12. Jones $L$, Walsh $K$, Wilcox $M$, Morgan P, Nichols J. The Covid19 pandemic: Important considerations for contact lens practitioners. Cont Lens Anterior Eye.2020 June; 43(3):196203. doi: 10.1016/j.clae.2020.03.012. Epub 2020 Apr 3.

13. American academy of optometry \& American Optometry association: In office disinfection of multipatient use diagnostic contact lenses. Available from:https://www.aaopt.org/docs/covid19/aao008

disinfectionofcontactlenseschart.pdf? Sfvrsn=a7f2a31_2. [Last accessed on 2020 Jun 24]

14. Zeri F and Naroo SA. Contact Lens practice in the time of COVID19. Cont Lens Anterior Eye 2020; 43:1935. doi: 10.1016/j.clae.2020.03.007. Epub 2020 Mar 19

15. Villahoz NG, Blasco BB,Villegas BV,Feijoo JG,Villalobos PA, Valle DD et al.. Novel Insights into the Transmission of SARS-CoV-2 through the Ocular Surface and its Detection in Tears and Conjunctival Secretions: Adv Ther. 2020 Oct; 37(10):4086-4095. doi: 10.1007/s12325-020-01442-7. women 